\title{
Analysis of the dc conductivity of the quasi-one-dimensional charge-density-wave conductor (fluoranthene) ${ }_{2} X$
}

\author{
P. H. Nguyen, \\ Physikalisches Institut und Bayreuther Institut für Makromolekülforschung (BIMF), Universität Bayreuth, \\ D-95440 Bayreuth, Germany \\ G. Paasch \\ Institut für Festkörperforschung und Werkstofforschung e.v., D-01171 Dresden, Germany \\ W. Brütting and W. Riess \\ Physikalisches Institut und Bayreuther Institut für Makromolekülforschung (BIMF), Universität Bayreuth, \\ D-95440 Bayreuth, Germany \\ (Received 10 August 1993)
}

\begin{abstract}
It has been shown recently that the dc conductivity (along the highly conducting axis) of fluoranthene radical cation salts exhibits the behavior of a quasi-one-dimensional conductor with a Peierls transition at about $180 \mathrm{~K}$ to a charge-density-wave ground state. In the high-temperature range fluctuations of the order parameter lead to a pseudogap in the electronic density of states, whereas below the phase transition the energy gap is BCS-like. To analyze the conductivity data, a simple band-structure model is used and related to optical data. In spite of the occurrence of the Peierls transition, it is assumed that threedimensional effects and fluctuations are strong enough for the conduction to be essentially due to electron-hole transport in bands and not polaronlike. Therefore the conductivity is determined simply by the Boltzmann equation with deformation-potential scattering. (a) This model yields a good fit of experimental data using the BCS-like gap below the transition and the pseudogap as determined from paramagnetic spin susceptibility above the transition. (b) It is possible to obtain the temperature dependence of the concentration, the mobility, the relaxation time, and the mean free path of carriers. (c) One can check a fundamental criterion for the applicability of the model. (d) We obtain from the fit parameters reasonable estimates of the quantities determining the deformation-potential scattering. Moreover, it is demonstrated that the theory can be used to determine the full temperature dependence of the gap/pseudogap directly from conductivity data.
\end{abstract}

\section{INTRODUCTION}

Depending on the dominating interaction (electronphonon or electron-electron interaction) quasi-onedimensional electron systems show instabilities leading to ground states with attracting properties such as e.g., bound order waves, charge-density waves (CDW's) or spin-density waves (SDW's). ${ }^{1,2}$ Originally observed in inorganic systems such as linear chain platinum compounds, several transition metal compounds, and the blue molybdenum bronzes, the Peierls transition to a CDW ground state has also been found in organic materials like tetrafluoro-tetracyanoquinodimethane (TTF-TCNQ). ${ }^{3}$

A detailed analysis of the Peierls instability in the quasi-one-dimensional organic $\mathrm{CDW}$ conductor (fluoranthene) ${ }_{2} X\left(X^{-}=\mathbf{P F}_{6}^{-}, \mathrm{AsF}_{6}^{-}\right.$, and $\left.\mathrm{SbF}_{6}^{-}\right)$has been presented recently, ${ }^{4}$ with special attention to the dc conductivity. The conductivity data were analyzed using the Boltzmann description in the relaxation-time approximation. The expression for the conductivity used in Ref. [4] involves some further approximations: the velocity and relaxation time are independent of the energy of carriers, and a phenomenological $T^{-B}(B \approx 1)$ temperature dependence of the relaxation time is used. The gap below the Peierls transition is assumed to have a BCS-like temperature dependence, whereas above the transitionaccording to measurements of the paramagnetic spin susceptibility ${ }^{5}$ - a weakly temperature-dependent pseudogap is used. In spite of the remarkable fit of conductivity data achieved in this way, there arise some new questions closely connected to one another.
At first, in a one-dimensional coupled electron-phonon system undergoing a Peierls transition, the lowest excitation will not be a purely electronic band-to-band excitation but a polaronic coupled electron-lattice distortion excitation. Although only little details have been worked out for the transport behavior of such excitations, it is clear that it can hardly be described by a quasiclassical kinetic equation such as the Boltzmann equation. Actually, the Peierls transition itself is already modified due to three-dimensional effects and fluctuations of the order parameter: ${ }^{6-8}$ one observes a lowering of the transition temperature and a modified temperature dependence of the gap below the transition, and there occurs a fluctuating pseudogap ${ }^{9}$ above the transition. It is trivial that these modifications are accompanied by a decrease of the energetic difference between coupled electron-lattice excitations and the simple electronic band-to-band excitations. Using the Boltzmann equation for the description of transport in such systems is implicitly based on the assumption that three-dimensional effects and fluctuations are not strong enough to prevent the occurrence of the transition, but at the same time are of such magnitude that the excitations determining the transport can be described effectively as simple single-particle excitations from the valence to the conduction band. It is just this assumption which will be examined here.

Further, it is not sufficient that experimental data can be well fitted by a given theoretical temperature dependence. The parameters following from the fit also must be in reasonable quantitative agreement with the assumed 
model. Also, basic conditions for the validity of the theory used must be fulfilled.

In this paper we describe the dc transport data of fluoranthene radical cation salts consistently within the above-mentioned quasiclassical picture. A model for the band structure containing a minimum of assumptions is used, and its main parameter is determined from optical data. First a temperature dependence of the gap is used, as determined in Ref. 4. The corresponding energy and temperature dependence of the velocity of charge carriers, and the correctly described energy and temperature dependence of the relaxation time due to deformationpotential scattering are used in the conductivity. In this way not only is a remarkable description of the experimental temperature dependence achieved, but the parameters also lie within a reasonable range; temperature dependences of the concentration, mobility, relaxation time, and mean free path of carriers can be determined, and a fundamental condition for the applicability of the Boltzmann description can be tested. Finally, we propose and test the possibility to determine directly the temperature dependence of the Peierls gap (below the transition) and the pseudogap (above the transition) from conductivity data. This is important insofar as the actual temperature dependence is more involved than, e.g., described by existing simple theoretical models such as the scaled mean-field dependence below the transition. ${ }^{8}$

\section{CRYSTAL STRUCTURE, CONDUCTIVITY AND ENERGY GAP IN (FA $)_{2} X$}

Fluoranthene radical cation salts $(\mathrm{FA})_{2} X$ are highly conducting crystals whose simple crystalline and molecular structures make them model systems for quasi-onedimensional organic conductors. The crystal structure ${ }^{10}$ is characterized by the presence of slightly dimerized donor stacks of planar fluoranthene molecules $(\mathrm{FA}=$ $\mathrm{C}_{16} \mathrm{H}_{10}$ ) piled in a zigzag manner along the crystallographic $a$ axis. Columns of monovalent anions $X^{-}$such as $\mathrm{PF}_{6}{ }^{-}, \mathrm{AsF}_{6}{ }^{-}$, or $\mathrm{SbF}_{6}{ }^{-}$separate the donor stacks in $b$ and $c$ directions perpendicular to the $\pi$-electron system of the molecule. The strong $\pi$-electron overlap along the stack axis together with the average charge transfer of 0.5 electrons per FA molecule allow the formation of a partly filled quasi-one-dimensional conduction band in the $a$ direction. The large average distance between the FA stacks and the directed $\pi$ orbitals are responsible for the strong anisotropy of many physical properties, for example the optical reflectivity ${ }^{11}$ and electrical conductivi$t^{4}$ parallel and perpendicular to the $a$ axis.

Transport ${ }^{4}$ and magnetic measurements ${ }^{5}$ show the existence of a metal-insulator phase transition (a Peierls transition) at a transition temperature $T_{P} \approx 180 \mathrm{~K}$, depending on the size of the counterion. Figure 1 shows the temperature dependence of the dc conductivity $\sigma_{\|}$of a $(\mathrm{FA})_{2} \mathrm{PF}_{6}$ crystal parallel to the $a$ axis. The phase transition can be clearly identified from the singularity in the derivative.

Meanwhile, it is well established by $\mathrm{x}$-ray investigations that this phase transition is driven by the electronphonon interaction leading to a $2 k_{F}$ instability. ${ }^{12}$ Conse-

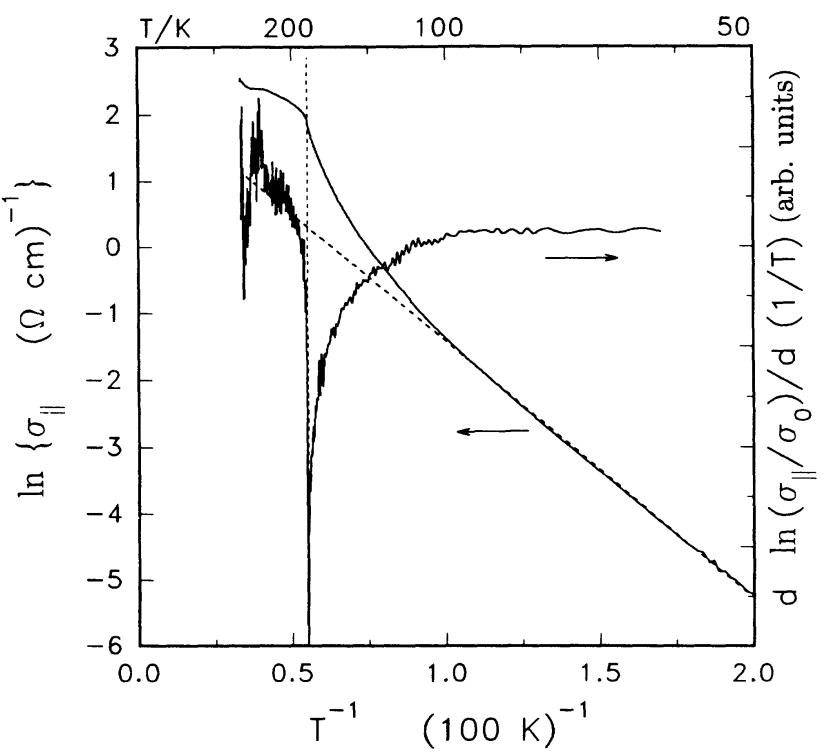

FIG. 1. Measured dc conductivity $\sigma_{\|}(T)$ vs the reciprocal temperature and logarithmic derivative, showing clearly the transition (from Ref. 4). Below about $100 \mathrm{~K}$ the thermally activated conductivity is measured with activation energies $\Delta(0)=60-90 \mathrm{meV}$ on different crystals.

quently the ground state is a CDW state. Indeed, in the temperature range below the transition, $(\mathrm{FA})_{2} X$ shows several collective transport properties such as nonlinear conductivity above a small sample-dependent threshold field, conduction noise in the nonlinear state, frequencydependent conductivity, and metastability phenomena. ${ }^{13-16}$ Contrary to the simple strictly one-dimensional mean-field picture, the Peierls gap does not vanish at the transition temperature, but above $T_{P}(180<T<300 \mathrm{~K})$ there exists a weakly temperature-dependent so-called pseudogap, as indicated by the nonmetallic temperature dependence of the conductivity in this temperature range, and by magnetic measurements. ${ }^{5,17}$ The pseudogap is attributed to pretransitional fluctuations and residual effects of the three dimensionality. ${ }^{6,7}$

Using a simplified description it has been shown ${ }^{4}$ that due to its high one dimensionality $(\mathrm{FA})_{2} X$ behaves over the entire temperature range investigated like a onedimensional semiconductor with a temperature dependent gap. As shown in Fig. 2, there is scaled BCS-like temperature dependence below the transition and, above it, a pseudogap which is consistent with magnetic measurements (for details, see Appendix A).

\section{THEORETICAL MODEL FOR BAND TRANSPORT IN THE QUASI-ONE DIMENSIONAL CONDUCTOR (FA) ${ }_{2} X$}

According to the experimental findings, it is assumed that three-dimensional effects and fluctuations are not strong enough to prevent the Peierls transition from occurring at $T_{P} \approx 180 \mathrm{~K}$, which is considerably below the mean field value $T_{p}^{\mathrm{MF}} \approx 400-600 \mathrm{~K}$. Here we will test the hypothesis that both effects can be sufficiently large at the same time, so that the electronic transport is essentially 


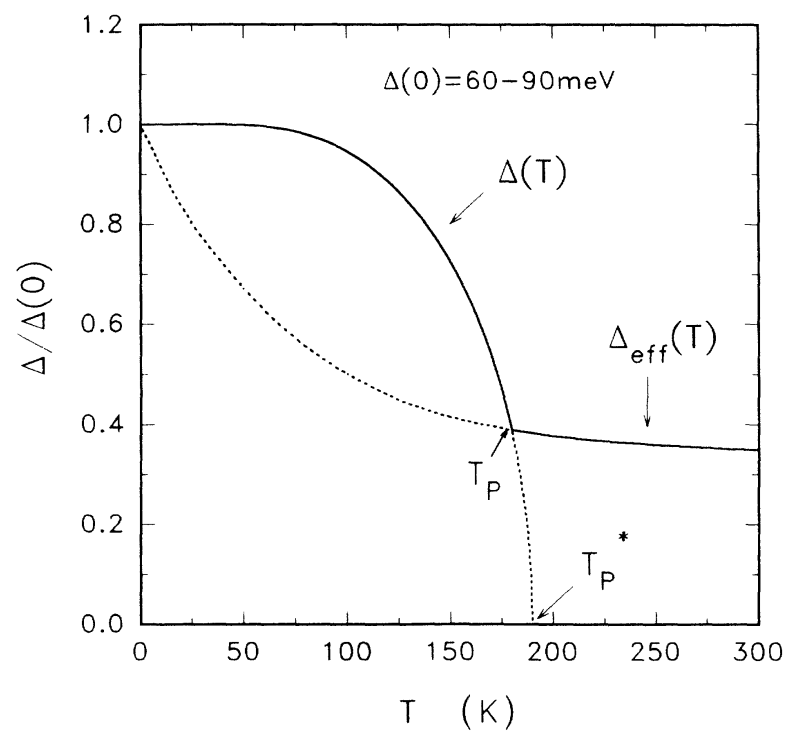

FIG. 2. Temperature dependence of the Peierls gap used to describe the conductivity data: Below $T_{P}$, the scaled theoretical mean-field dependence is used. Above $T_{p}$ it is the effective gap deduced from the paramagnetic spin susceptibility (from Ref. 4).

that of a highly anisotropic conventional threedimensional system, i.e., transport of thermally excited carries rather than a polaronlike transport.

\section{A. Band structure}

Since there is practically no direct experimental or theoretical model of the band structure, we use a simple reasonable tight-binding (TB) model (other TB models ${ }^{18}$ have also been tested; though essentially different after determination of the main parameter from optical data, the numerical results for the conductivity are comparable). The overlap of the total $\pi$-electron system of the FA molecules occurs in the chain direction perpendicular to the molecular plane, and is characterized by the nearestneighbor matrix element $t_{\|}$. Neglecting dimerization, the simplest TB band is $E_{k}^{(0)}=-2 t_{\|} \cos (k a / 2)$ for $|k| \leq 2 \pi / a,{ }^{18}$ where $a=6.61 \AA$ is the lattice constant of the dimerized lattice. This band would have a $\frac{3}{4}$ filling since there are two molecules in the elementary cell, four states (spin), and one charge is transfered to the anion. Actually there is already dimerization at room temperature. With dimerization (parameter $2 \Delta_{\|}$for convenience, as introduced in Ref. 18), one has a lower completely filled and an upper half filled subband (Fig. 3):

$$
E_{k \pm}^{(d)}= \pm \sqrt{\left(E_{k}^{(0)}\right)^{2}+\left(2 \Delta_{\|}\right)^{2}}, \quad|k| \leq \pi / a,
$$

with

$$
E_{k}^{(0)}=-2 t_{\|} \cos (k a / 2) .
$$

The two new subbands $E_{k \pm}^{(d)}$ are separated by an energy gap of $4 \Delta_{\|}$at $\pi / a$. The upper subband is occupied from $\pi / 2 a$ to $\pi / a$, and the Fermi energy is

$E_{F}=\sqrt{2} t_{\|} \sqrt{1+2\left(\Delta_{\|} / t_{\|}\right)^{2}}=\sqrt{2} t_{\|}+O\left(\left(\Delta_{\|} / t_{\|}\right)^{2}\right)$

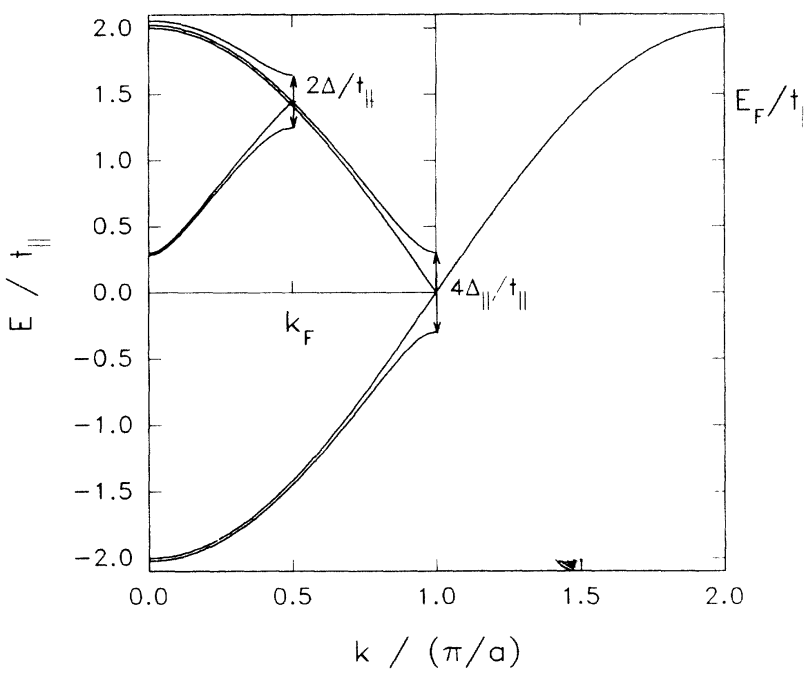

FIG. 3. Band-structure model of $(\mathrm{FA})_{2} X$ with a gap $4 \Delta_{\|}$due to dimerization, and a gap $2 \Delta$ (Peierls gap/pseudogap) due to the $2 k_{F}$ instability.

and $\varepsilon_{F} \equiv E_{F}-2 \Delta_{\|}$is the Fermi energy relative to the bottom of the subband $E_{k+}^{(d)}$.

Following Ref. 11, we use the plasma frequency determined from optical data, $\hbar \omega_{p \|} \approx 1.5 \mathrm{eV}$ (small damping $\left.\hbar / \tau_{p} \approx 0.1 \mathrm{eV}\right)$ to obtain $t_{\|}$. Contrary to Ref. 11, the correct connection

$\omega_{p \|}^{2}=\frac{e^{2} N / a}{\varepsilon_{0} m_{\mathrm{opt}}}, \frac{1}{m_{\mathrm{opt}}}=\frac{2 a}{\pi \hbar^{2}}\left|\frac{d E_{k+}^{(d)}}{d k}\right|_{k_{F}=\pi / 2 a}$

is used ( $N$ is the number of FA chains per unit area, $N=2 / b c, b=12.57 \AA$, and $c=14.77 \AA$ ). Considering only the term linear in $\Delta_{\|}$, one obtains $E_{F}=\varepsilon_{F}+2 \Delta \| \approx \sqrt{2} t_{\|} \approx 0.55 \mathrm{eV}$ and accidentally $m_{\text {opt }} \approx m_{0}$. The distance from the top of the band $E_{k+}^{(d)}$ to the Fermi energy - the Fermi energy of holes-is 0.23 $\mathrm{eV}$. If a gap exists at the temperature at which the optical measurements were made - contrary to the assumption leading to (3) - one arrives at essentially the same result with $m_{\mathrm{opt}} \rightarrow m_{0}{ }^{11}$

The occurrence of the Peierls gap or pseudogap $2 \Delta$ at $k_{F}=\pi / 2 a$ can be included, leading to $E=E(k)$ as the solution of

$$
\left|\begin{array}{cc}
E_{k+}^{(d, u)}-E & \Delta \\
\Delta & E_{k+}^{(d, d)}-E
\end{array}\right|=0, \quad|k| \leq \pi / 2 a,
$$

where

$$
\begin{aligned}
& E_{k+}^{(d, u)}=2 \sqrt{\left(t_{\|} \cos (k a / 2)\right)^{2}+\Delta_{\|}^{2}}, \\
& E_{k+}^{(d, d)}=2 \sqrt{\left(t_{\|} \sin (k a / 2)\right)^{2}+\Delta_{\|}^{2}},
\end{aligned}
$$

follows from folding $E_{k+}^{(d)}$.

According to Sec. II and Fig. 2, for the temperaturedependent gap $\Delta(T)$ in (4) for $T<T_{P}$ we use a scaled Peierls gap depending BCS-like on $T^{4}$ On the other hand, above $T_{P}$ at least up to room temperature there 
remains a pseudogap due to three dimensionality and fluctuations, with a magnitude larger than $k_{B} T$. Then only the band structure in the vicinity of the gap (at $k=k_{F}$ ) is of interest. In the parabolic approximation Eq. (4) yields the velocity of carriers $v(\varepsilon)$ [ $\varepsilon$ is relative to the band extremum at $\pi / 2 a: \varepsilon \equiv E-\left(E_{F}+\Delta\right)$ ], the density of states $\mathcal{D}(\varepsilon)$, and the effective mass $m_{\text {eff }}$ [from $\left.v=\hbar(k-\pi / 2 a) / m_{\mathrm{eff}}\right]$ :

$$
\begin{aligned}
& v(\varepsilon)=\frac{a}{2 \hbar} E_{F}\left(\frac{2 \varepsilon}{\Delta}\right)^{1 / 2}, \\
& \mathcal{D}(\varepsilon)=\frac{2}{\pi \hbar v(\varepsilon)}, \\
& \frac{1}{m_{\mathrm{eff}}}=\frac{1}{\Delta}\left(\frac{a E_{F}}{2 \hbar}\right)^{2} .
\end{aligned}
$$

All three quantities contain only $E_{F}$, which (contrary to $\varepsilon_{F}=E_{F}-2 \Delta_{\|}$) up to linear order in $\Delta_{\|}$is determined directly from $\hbar \omega_{p \|}$. Thus the knowledge of $\Delta_{\|}$itself is not necessary. With $E_{F}=0.55 \mathrm{eV}$ and $0.02<\Delta / \mathrm{eV}<0.09$ (Fig. 2), the effective band mass near the gap follows from (5) as $m_{\text {eff }}=0.2 \ldots 0.5 m_{0}$. This quantity will be of interest below in discussing the mobility.

\section{B. de conductivity and related quantities}

The approximation $\sigma_{\| t}(T) \sim T^{-B}\left[\exp \left(\Delta / k_{B} T\right)+1\right]^{-1}$ used by several authors ${ }^{4,14,19}$ to describe the conductivity in CDW systems is based essentially upon the following assumptions: (a) transport by electrons and holes in the conduction and valence bands, respectively [corresponding to our $E(k)$ ]; (b) applicability of the Boltzmann equation within (c) the relaxation-time approximation, (d) constant carrier velocity, (e) energy-independent relaxation time, and (f) a phenomenological $T^{-B}(B \approx 1)$ dependence of the relaxation time (the citation ${ }^{4}$ of this temperature dependence does not correspond to the semiconducting state of the material). Since approximations (d) - (f) are not generally valid, we will replace them by appropriate relations. In addition, parameters obtained from fitting experimental data will be used to check to some extent the basic assumptions (a)-(c).

Although we assume a sufficient effect of three dimensionality and fluctuations, the transport is essentially a highly anisotropic electron-hole transport in bands. The conductivity in the chain direction is then determined mainly by the quasi-one-dimensional band structure discussed above. It is given by

$$
\sigma=2 e^{2} N \int_{\Delta}^{\infty} d E \mathscr{D}(E) \tau(E) v^{2}(E)\left(-\frac{\partial f^{0}}{\partial E}\right),
$$

including both electron and hole contributions by assuming the two bands are near the gap symmetric with respect to the Fermi energy. Due to the factor $\left(-\partial f^{0} / \partial E\right)$, this is the case for $\Delta \geq k_{B} T$, and the bands can be approximated parabolically leading to expressions (5) for the velocity and density of states (and to the upper limit of the integral for the same reason).

The evaluate (6) one needs finally the energy and tem- perature dependence of the relaxation time. Scattering by charged counterions will make a negligible contribution due to their arrangement in columns between the FA chains, as shown in Ref. 20. According to our basic assumption there remains the scattering by acoustic and nonpolar optical phonons. Due to the large number of phonon branches which have been neither measured nor calculated, we prefer here to use the deformationpotential method. The one-dimensional version of the textbook expressions ${ }^{21}$ is obtained easily. The resulting energy and temperature dependence is given [using the density of states (5)] by

$$
\tau(E)=\frac{\mathcal{B}}{\mathcal{D}(E) T},
$$

with

$$
k_{B} \mathcal{B} \equiv \frac{\hbar p_{a, o}}{2 \pi a}, p_{a} \equiv \frac{M c^{2}}{E_{a}^{2}}, \quad p_{o} \equiv \frac{M \omega_{o}^{2}}{E_{o}^{2}} .
$$

As usual, both processes yield the same dependence. In (7), $M$ is the molecular mass (of the FA molecule), $c$ the velocity of sound, $\omega_{o}$ the nonpolar optical-phonon frequency, $E_{a}$ the acoustic deformation potential, and $E_{o}$ the optical deformation potential. Using (5) and (7), one can evaluate (6) analytically to obtain

$$
\sigma(T)=C \frac{\ln \left(1+e^{-\Delta(T) / k_{B} T}\right)}{\Delta(T)}, C \equiv \frac{e^{2}}{h} a N E_{F}^{2} p_{a, o},
$$

Of course, using the full expression for $E(k)$ from (4) one obtains $D(E), v(E)$, and $\tau(E)$ without the parabolic approximation, and (6) can be integrated numerically. Though this procedure is possible, it provides only minor corrections in fitting the data as far as the condition $\Delta \geq k_{B} T$ is fulfilled. Thus we prefer the clear analytical dependence of (8).

The quantity which is actually measured is the resistivity of a sample of length $l$ and cross section $S$. The data will then be fitted with

$$
R(T)=r \frac{\Delta(T)}{\ln \left(1+e^{-\Delta(T) / k_{B} T}\right)}, r \equiv \frac{l}{S} \frac{h}{e^{2} N a E_{F}^{2} p_{a, o}} .
$$

For the carrier density $n=2 N \int d E \mathscr{D}(E) f^{0}(E)$, the onedimensional case yields $[\Delta=\Delta(T)]$

$$
n=2\left(\frac{2}{\pi}\right)^{1 / 2} \frac{N}{a} \frac{\sqrt{k_{B} T \Delta}}{E_{F}} F_{-1 / 2}\left(-\Delta / k_{B} T\right),
$$

with

$$
F_{-1 / 2}(y)=\frac{1}{\sqrt{\pi}} \int_{0}^{\infty} \frac{d x x^{-1 / 2}}{1+e^{x-y}} .
$$

Together with (8) and (9), the electron and hole mobilities $\mu=\sigma / 2$ en can be calculated as

$$
\mu=\frac{l}{S r} \frac{1}{4}\left(\frac{\pi}{2}\right)^{1 / 2} \frac{a}{e N} \frac{E_{F}}{\Delta \sqrt{\Delta k_{B} T}} \frac{\ln \left(1+e^{-\Delta / k_{B} T}\right)}{F_{-1 / 2}\left(-\Delta / k_{B} T\right)} .
$$


Further, the mean relaxation time $\bar{\tau}=\mu m_{\text {eff }} / e$ is, with the effective mass (5),

$\bar{\tau}=\frac{l}{S r}\left(\frac{\pi}{2}\right)^{1 / 2}\left(\frac{\hbar}{e}\right)^{2} \frac{1}{N a} \frac{1}{E_{F} \sqrt{\Delta k_{B} T}} \frac{\ln \left(1+e^{-\Delta / k_{B} T}\right)}{F_{-1 / 2}\left(-\Delta / k_{B} T\right)}$,

and the mean free path $\lambda=v_{\mathrm{th}} \bar{\tau}$ is given by

$$
\lambda=\frac{l}{S r} \frac{1}{2}\left(\frac{\pi}{2}\right)^{1 / 2} \frac{\hbar}{e^{2} N \Delta} \frac{\ln \left(1+e^{-\Delta / k_{B} T}\right)}{F_{-1 / 2}\left(-\Delta / k_{B} T\right)},
$$

with $v_{\mathrm{th}}=\sqrt{k_{B} T / m_{\text {eff }}}$ also containing the effective mass. Finally, a fundamental condition for the applicability of the Boltzmann equation in the relaxation-time approximation is

$$
k_{B} T \gg \hbar / \bar{\tau} .
$$

With (13), one has (see also Appendix B)

$$
\begin{aligned}
k_{B} T \frac{\bar{\tau}}{\hbar}= & \frac{l}{S r}\left[\frac{\pi}{2}\right]^{1 / 2} \frac{\hbar}{e^{2}} \frac{1}{N a E_{F}}\left(\frac{k_{B} T}{\Delta}\right)^{1 / 2} \\
& \times \frac{\ln \left(1+e^{-\Delta / k_{B} T}\right)}{F_{-1 / 2}\left(-\Delta / k_{B} T\right)} .
\end{aligned}
$$

In the following, these expressions are applied to describe the experimental data of the dc conductivity of $(\mathrm{FA})_{2} X$.

It should be mentioned that (for quasi-one-dimensional conductors such as TTF-TCNQ) Conwell ${ }^{22}$ derived an expression for $\mu$ in the acoustic-phonon scattering case that is also proportional to $\Delta^{-3 / 2} T^{-1 / 2}$ like (12) but neglects the remaining $T$ dependence $\left[\ln (\cdots) / F_{-1 / 2}(\cdots)\right]$ arising from averaging the relaxation time and from the carrier density. Also conditions (15) and (16) for the applicability of the Boltzmann equation have not been considered in her work.

\section{RESULTS}

\section{A. Fit of the de-conductivity data}

The description given in Sec. III will now be used to analyze the measured resistivity of four $(\mathrm{FA})_{2} \mathrm{PF}_{6}$ samples. Here we assume that the gap $\Delta(T)$ is given sufficiently accurately by the temperature dependence (Fig. 2) explained in Sec. II. Then the theoretical expression (9) contains merely one free parameter $r$. The reliability of such a description can be checked by considering the dependence (Fig. 4 for $T<T_{P}$ )

$\ln (R / \Omega)$

VS

$$
x=\ln \left\{(\Delta / \mathrm{meV}) / \ln \left(1+\exp \left[-\Delta / k_{B} T\right]\right)\right\},
$$

which will be approximated by linear regression as

$$
\ln (R / \Omega)=A+B x \text {. }
$$

In the case where $B=1$, Eq. (9) [with the assumed $\Delta(T)$ ] describes the observed temperature dependence exactly.

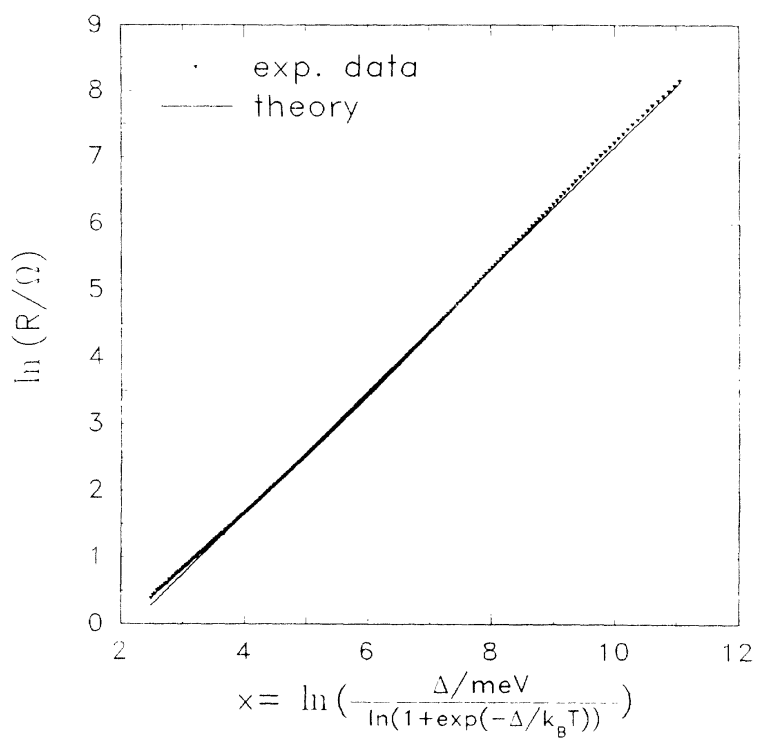

FIG. 4. Linear regression according to Eq (18), using the gap shown in Fig. 2 (for $T<T_{P}$ ) for $\Delta(T)$.

The example in Fig. 4 shows that there is indeed a linear dependence with a $B$ value near unity. Fit parameters for four samples are given in Table I for $T<T_{P}$. The small deviation from $B=1$ can either be due to the assumptions and approximations made to obtain the dependence used for $\Delta(T)$ or to the approximations needed to derive (9). The experimental resistance values are then approximated by $10^{A} X^{B}\left(X \equiv 10^{x}\right)$. A similar procedure is applied to the temperature range above the Peierls transition. An example for the resulting fit is shown in Fig. 5. It can be seen that Eq. (9) indeed contains all characteristics of the measured temperature dependence of the electrical resistance over more than eight orders of magnitude.

As mentioned above it is not sufficient for a formula [like (9) in our case] to describe well the functional dependence observed; the parameters determined from the fit (here $r$ ) also must be of reasonable magnitude corresponding to the model used. Though the deviation of $B$ from unity is small (Table I), it has to be taken into account in determining $r$. This is done on the following way. Since (9) with (18) has the form $R=r X=r X^{1-B} X^{B}$, and the fit is achieved with $10^{A} X^{B}$, the deviation $(1-B)$ of the exponent is averaged with respect to the temperature in the range considered. Then one obtains

$$
\begin{aligned}
& \frac{R}{\Omega}=\frac{r}{\Omega / \mathrm{meV}}{\overline{\left(X^{1-B}\right)^{T}} X^{B}=10^{A} X^{B},}^{\frac{r}{\Omega / \mathrm{meV}}=\frac{10^{A}}{\left(X^{1-B}\right)^{T}} .}
\end{aligned}
$$

TABLE I. Parameters $A$ and $B$ determined from fitting equation (18) for four different samples.

\begin{tabular}{lrrrr}
\hline \hline Sample & 1 & \multicolumn{1}{c}{2} & \multicolumn{1}{c}{3} & \multicolumn{1}{c}{4} \\
\hline$B$ & 0.915 & 0.991 & 0.914 & 0.938 \\
$A$ & -1.996 & -1.758 & -1.324 & -1.537 \\
\hline
\end{tabular}




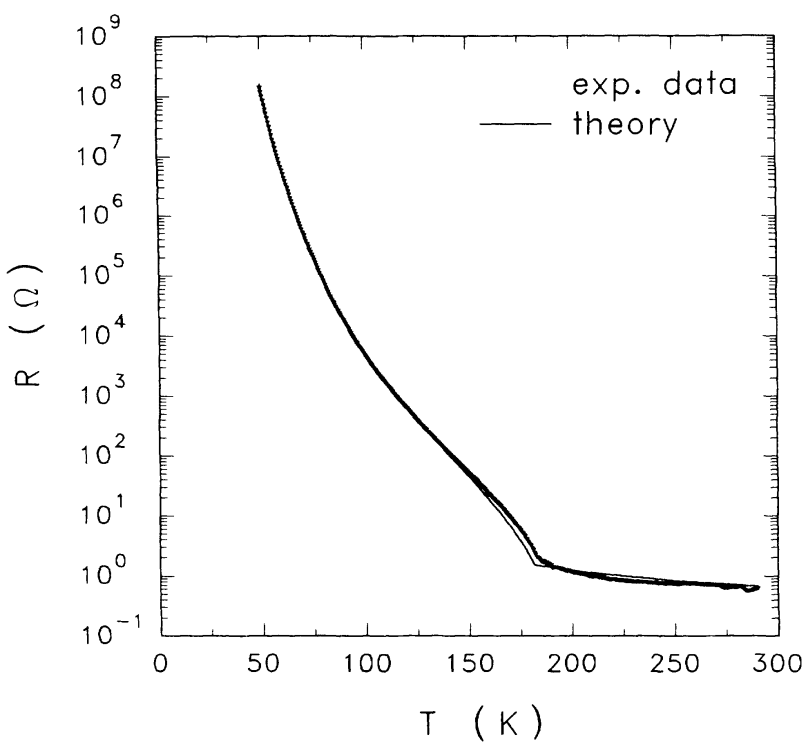

FIG. 5. Temperature dependence of the electrical resistance as calculated from Eq. (9), compared to the experimental data. Good agreement is found over the whole temperature range below and above the transition.

The values for $r$ are given in Table II. A typical value for ${\overline{\left(X^{1-B}\right.}}^{T}$ is 3.4 (for the sample of Fig. 4). The main problem is that the length $l$ and cross section $S$ of the sample entering the prefactor $r$ are effective values, the determination of which is fraught with many uncertainties. The geometry independent constant $C$ in (8) is thus affected by this uncertainty. The variation of $C$ between the samples (Table II) can be due partly to this effect. However, in addition different crystal-growth conditions and defects can be responsible for the variation of the absolute conductivity values.

\section{B. Calculation of related transport quantities}

We are now able also to calculate the temperature dependence of the other quantities related to the conductivity. At first, we mention that the variation of the conductivity over eight orders of magnitude arises mainly from the dependence of the carrier density (10) and (11) shown in Fig. 6 . Consequently the ratio $\ln (\ldots) / F_{-1 / 2}(\ldots)$ occurring in (12)-(14) and (16) varies only between 1 at low temperatures and approximately 1.06 at $300 \mathrm{~K}$, but it shows a very pronounced structure

TABLE II. Length and cross section for the four samples leading together with the quantity $r(19)$ to the constant $C$ in the conductivity (8).

\begin{tabular}{lcccc}
\hline Sample & 1 & 2 & 3 & 4 \\
\hline$l / \mathrm{mm}$ & 0.6 & 0.9 & 0.9 & 0.6 \\
$S / \mathrm{mm}^{2}$ & $(0.125)^{2}$ & $(0.2)^{2}$ & $(0.16)^{2}$ & $(0.15)^{2}$ \\
$r / \frac{\Omega}{\mathrm{eV}}$ & 3.0 & 15.75 & 13.25 & 11.57 \\
$C / \frac{\mathrm{eV}}{\Omega \mathrm{cm}}$ & 128.0 & 14.28 & 26.53 & 23.05 \\
\hline
\end{tabular}

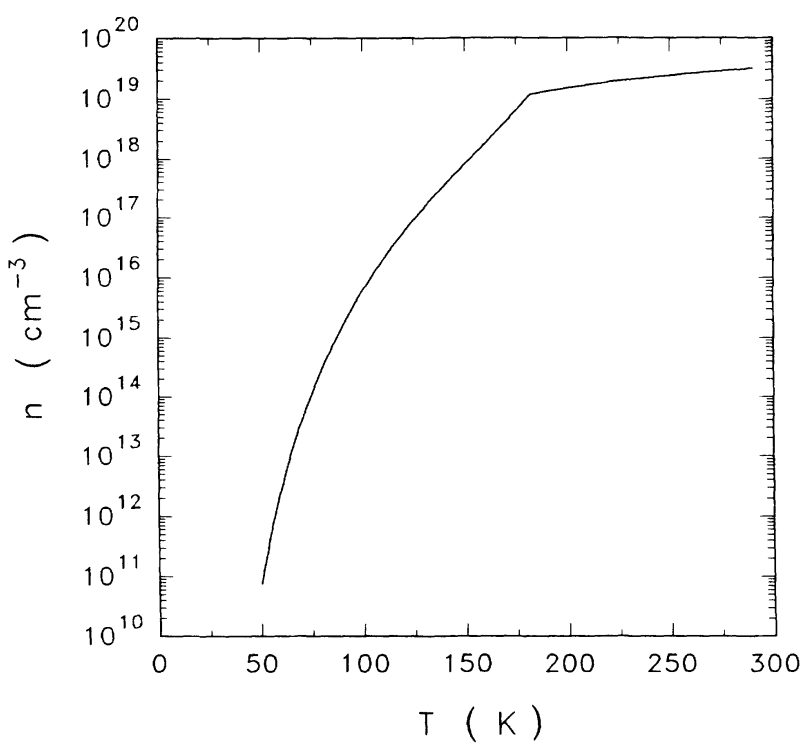

FIG. 6. The theoretical (intrinsic) electron density in the conduction band. The gap of Fig. 2 is used. The carrier concentration at room temperature is a factor of 50 lower than the carrier density $n=2 /(a b c)=1.6 \times 10^{21} \mathrm{~cm}^{-3}$, following from simple chemical considerations.

at the transition temperature $T_{P}$, which also will be seen in $\bar{\tau}, \mu, \lambda$, and (16). Figures $7(\mathrm{a})-7(\mathrm{c})$ show the mean relaxation time, the mobility, and the mean free path, respectively. The strong change in the slope of all three quantities at the transition temperature $T_{P}$ arising from the change in the slope of the gap (Fig. 2) is remarkable. This change is still finite, since the CDW gap does not vanish at the transition temperature but leads into the pseudogap. Further, since according to (12)-(14) all three quantities have a common dependence on $\ln (\ldots) / F_{-1 / 2}(\ldots)$, the variation over nearly eight orders of magnitude seen in the conductivity is canceled out, and the temperature dependence of $\bar{\tau}, \lambda$ and $\mu$ is mainly determined by the dependence on $\Delta^{\alpha}(T) T^{\beta}$ with different exponents $\alpha$ and $\beta$. This dependence is smallest in the case of the relaxation time, where $\Delta$ (decreasing with $T$ ) and $T$ occur simply as the square root of the product. But $\mu$ and $\lambda$ also vary in the temperature range considered, less than one order of magnitude. This small dependence of all three quantities on temperature can be explained as follows. The $T^{-1}$ dependence of the deformation-potential scattering (7) is canceled in the conductivity due to the energy integration in (6), leading to the dependence (8). On the other hand, the $T^{-1}$ dependence itself arises due to both the one dimensionality and the equipartition assumption for the phonons. The latter is indeed justified in the case considered here, since the temperature interval $k_{B} T$ is from 4.3 to $26 \mathrm{meV}$, whereas the relevant phonon energy is estimated to be sufficiently smaller (about $2 \mathrm{meV}$; see below). The reliability of the absolute numbers of all three quantities is connected with condition (16) for the applicability of the Boltzmann equation. (According to Appendix B, one should be aware of the fact that the conditions for $\mu$ and the mean free path contain the effective mass, which is 

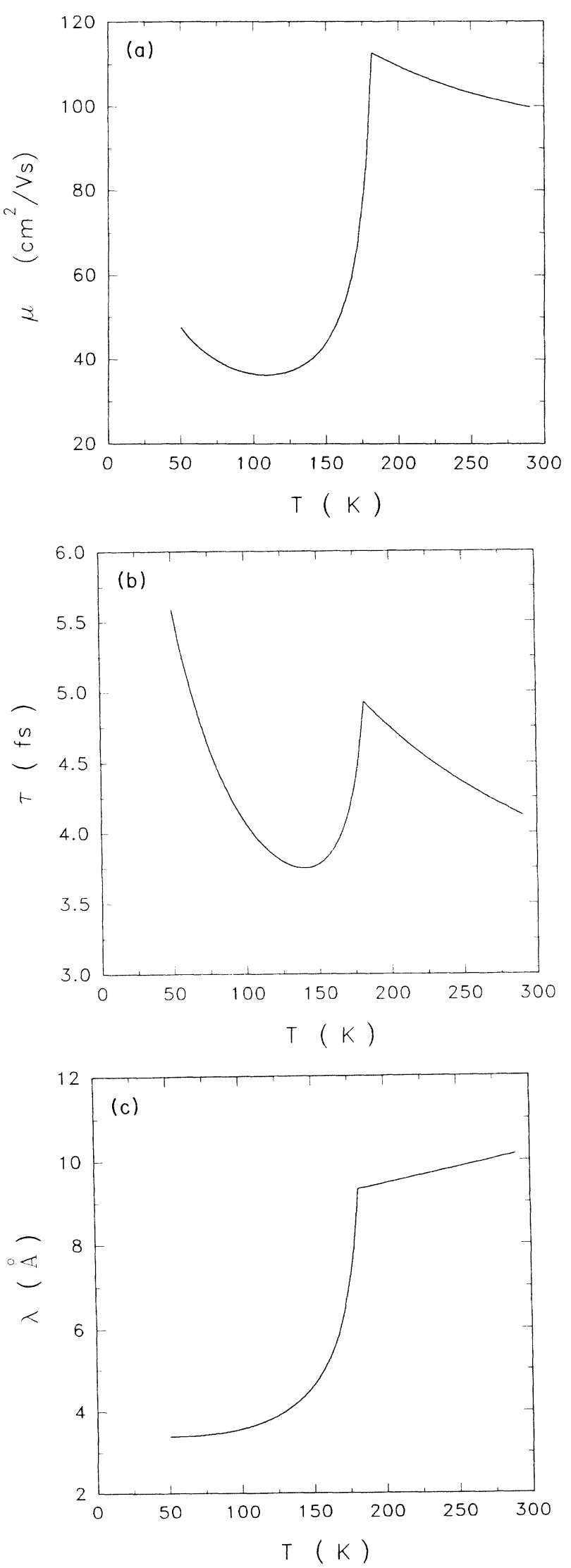

FIG. 7. Electron mobility (a), mean relaxation time (b), and mean free path (c) following from the fit of the experimental conductivity data using the gap shown in Fig. 2. small in our case). Quantity (16) has to obey condition (15). Figure 8 shows that the variation again is small over the entire temperature interval, but condition (15) is not fulfilled; this must be attributed to uncertainties in the absolute value of the conductivity, which is difficult to determine for the samples investigated. In spite of the uncertainties about condition (15), the good agreement between the measured resistivities and those described by (9) with the assumed temperature dependence of the gap strongly supports the model presented here.

At the end of this section another consequence of the fitted value $r$ will be discussed. According to (7) and (9), from the fit parameter $r$ one obtains the quantity

$$
M c^{2}=\left(\frac{E_{a}}{E_{F}}\right)^{2} \frac{l h}{r S e^{2} N a},
$$

characterizing the acoustic phonons. The difference from the nonpolar optical phonons results essentially from folding the phonon spectrum into the smaller Brillouin zone. Due to the relation for the acoustic deformation potential $E_{a}=\left(\frac{2}{3}\right) E_{F}$ (Ref. 23) (resulting from long-range Coulomb screening), neither $E_{F}$ nor $E_{a}$ are needed explicitly. Using $r \approx 3 \Omega / \mathrm{eV}$, as obtained above we get $M c^{2} \approx 0.2 \mathrm{eV}$, lying a little below the values typical for usual inorganic materials. ${ }^{21}$ With the mass of the $\mathrm{C}_{16} \mathrm{H}_{10}$ molecule $(202 \mathrm{u})$ this results in a sound velocity of $c \approx 310$ $\mathrm{m} / \mathrm{s}$, and finally the highest longitudinal phonon frequency will be approximately $\hbar \omega_{0} \approx \hbar c \pi / a \approx 1 \mathrm{meV}$. The values estimated here can be a little too small for the following reasons. First, there is a considerable uncertainty in the $r$ values (see above), and also in the geometrical factors sample length $l$ and sample cross section $S$. Further, it is not clear whether the relation $\left(E_{a} / E_{F}\right)=\frac{2}{3}$ is exactly valid for the system considered here. On the other hand, due to the high molecular mass one indeed has to expect low-lying phonons $\left(\omega_{0} \propto M^{-1 / 2}\right)$. Finally, the intermolecular $\pi$ overlap of the $\mathrm{C}$ atoms occurs in this

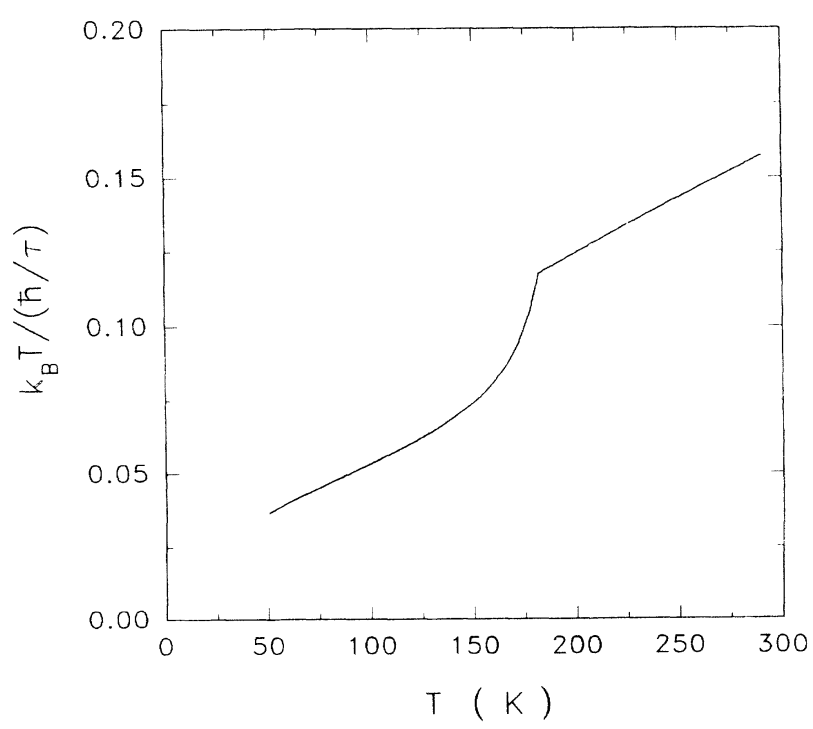

FIG. 8. The quantity $k_{B} T /(\hbar / \bar{\tau})$ occurring in conditions (15) and (16). For a comment on the absolute value, see the text. 
system perpendicular to the intramolecular $\pi$ overlap, and therefore more closely resembles a $\sigma$ binding with a large intramolecular spacing of $3.3 \AA$, contrary to the inplane carbon-carbon distance of about $1.4 \AA$ leading, e.g., in $(\mathbf{C H})_{n}$ to the high value $\hbar \omega_{0} \approx 0.1 \mathrm{eV} \cdot{ }^{24}$ For the latter two reasons small values of $c$ and $\omega_{0}$ must be expected.

\section{Determination of the temperature dependence of the gap from conductivity data}

Up to now we used the temperature-dependent gap $2 \Delta(T)$ as described in Sec. II and Appendix A. However, the actual $T$ dependence has been neither determined experimentally nor calculated taking into account with sufficient accuracy effects beyond the mean-field approximation. Below the transition temperature $T_{p}$, the scaled BCS-like mean-field dependence is used. Above $T_{p}$ pretransitional fluctuations lead to the formation of a pseudogap at the Fermi energy. The pseudogap actually used in fitting the conductivity in Refs. 4,17 , and 19, and earlier in this paper, is an effective gap $\Delta_{\text {eff }}(T)$ as determined from paramagnetic spin susceptibility (cf. Appendix A), which is an equilibrium quantity. Whether this is also the transport gap is not clear for two reasons. First, localized and delocalized states near the band edge affect the conductivity and the spin susceptibility differently. Second-keeping in mind the origin of the pseudogap from fluctuations - we must expect the ratio between the mean free path and the correlation length of the fluctuations to be important.

Due to these quite different uncertainties connected to the temperature dependence of the Peierls gap below the transition temperature, and the pseudogap/effective gap above as used earlier in fitting the conductivity data, we now propose to proceed in just the reverse manner: There is the Peierls gap below $T_{P}$ and above $T_{P}$ the pseudogap is replaced by an effective "transport" gap. The total dependence $\Delta(T)$ is the quantity to be determined. The theoretical description of the conductivity outlined in Sec. III B must be regarded as sufficiently accurate to be used for determining this unknown gap from the measured conductivity data under the following conditions. (a) There is actually a dominance of single-particle transport, which (b) can be described by the Boltzmann equation [condition of a sufficiently large relaxation time (Appendix B)]. (c) The transversal band width is negligible, and (d) the condition $\Delta(T) \geq k_{B} T$ is fulfilled. Finally, (e) phonon scattering will be the dominant process. According to the data given above, all these conditions seem to be realized sufficiently well. It can then be seen from Eqs. (8) and (9) that a given temperature dependence of the conductivity (resistivity) allows the determination of the temperature dependence $\Delta(T)$, provided that one has a reliable value for the gap at one temperature. We consider two possibilities. (A) the conductivity itself yields such information, with the activation energy following from the strictly linear dependence of $\ln (\sigma)$ vs $T^{-1}$ at low temperatures (100-50 K); for even lower temperatures the number of excited electrons and holes in the bands becomes too small, and other mechanisms will dominate the transport. On the other hand (B) one can also use the gap above $T_{p}$ somewhere in the region 200-300 K, as determined from paramagnetic spin susceptibility, although its connection with the transport gap is not quite clear. Figure 9 shows the temperature dependence of the gap determined in these ways in comparison with that used before (the scaled mean field behavior below $T_{P}$ and above the dependence from the paramagnetic spin susceptibility). In case (A) the constant in (9) is $r=3 \Omega / \mathrm{eV}$, and in case (B) it is $r=4.5 \Omega / \mathrm{eV}$. These values coincide with those used above for some estimates which need not be repeated here. Although the values of $r$ arising from the two approaches differ (apparently) considerably, the corresponding two gap functions differ over the entire temperature range from 50 to $300 \mathrm{~K}$ by less than $5 \mathrm{meV}$, as seen in Fig. 9. The decrease of the gap below $50 \mathrm{~K}$ in both cases is not significant, since for this temperature the description of (8) and (9) is no longer valid, as already mentioned. Further, between $50 \mathrm{~K}$ and $T_{P}$ the smooth decrease of the gap resembles the scaled mean-field dependence, but the strongest decrease is shifted a little to higher temperature. The transition from the Peierls gap to the effective pseudogap is well pronounced but not sharp. The dependence above $200 \mathrm{~K}$ exhibits some irregularities arising from the conductivity data itself; it should be mentioned that the crystals have been grown at $250 \mathrm{~K}$, and there may be some aging effects in the conductivity measured above this temperature.

The determination of the temperature dependence of the gap - the Peierls gap (below $T_{p}$ ) and the effective gap describing the influence of the pseudogap on the transport above the transition-directly from the conductivity data, as demonstrated here, has the advantages that the data used are relatively simple to obtain, and the result-

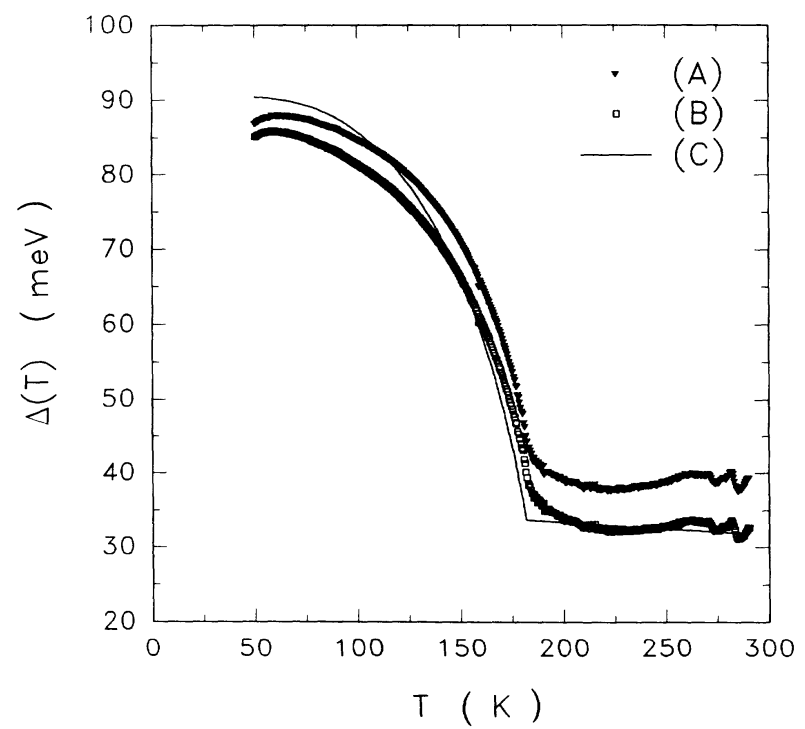

FIG. 9. Temperature dependence of the gap as deduced from the conductivity data with the two procedures $(A)$ and $(B)$ described in the text. The difference between the two curves is less than $5 \mathrm{meV}$. For comparison, the gap used earlier (Fig. 2) is also shown (C). 
ing gap contains all the effects which can hardly be described theoretically with sufficient accuracy.

\section{ACKNOWLEDGMENTS}

We thank M. Schwoerer for helpful discussions, and J. Gmeiner for growing the crystals. This work was supported by BASF/BMFT and Fonds der Chemischen Industrie.

\section{APPENDIX A}

The temperature dependence of the gap (Fig. 2) used in Sec. IV A to fit the experimental conductivity data is obtained from rather different information about the system above and below the transition temperature. In the mean-field theory, the Peierls gap $\Delta_{\mathrm{MF}}(T)$ below the transition temperature $T_{P}^{\mathrm{MF}}\left[\Delta_{\mathrm{MF}}\left(T_{P}^{\mathrm{MF}}\right)=0\right]$ of a strictly onedimensional system is determined ${ }^{7,25}$ by the implicit equation

$$
\frac{1}{\lambda}=\int_{0}^{\varepsilon_{B}} \frac{d \varepsilon}{\sqrt{\varepsilon^{2}+\Delta_{\mathrm{MF}}^{2}}} \tanh \left(\frac{\sqrt{\varepsilon^{2}+\Delta_{\mathrm{MF}}^{2}}}{k_{B} T}\right),
$$

where $\lambda$ is the dimensionless electron-phonon coupling constant. For $T=0$ and $T_{P}^{\mathrm{MF}}$ (A1) implies

$k_{B} T_{P}^{\mathrm{MF}}=1.14 \varepsilon_{B} \exp (-1 / \lambda)$,

$\Delta_{\mathrm{MF}}(0)=1.76 k_{B} T_{P}^{\mathrm{MF}}\left\{1+\frac{1}{2}\left[\sqrt{1+\Delta_{\mathrm{MF}}^{2}(0) / \varepsilon_{B}^{2}}-1\right]\right\}$.

Formally $2 \varepsilon_{B}$ is the interval around the Fermi energy, where for zero gap the dispersion relation can be linearized. Usually one replaces $\varepsilon_{B}$ with $\varepsilon_{F}$ and assumes further $\varepsilon_{F} \gg \Delta_{\mathrm{MF}}(0)$, leading to the connection

$$
\Delta_{\mathrm{MF}}(0)=1.76 k_{B} T_{P}^{\mathrm{MF}}
$$

and a dependence $\Delta_{\mathrm{MF}}(T)$ as in the BCS theory. However, the mean-field theory neglects thermodynamic fluctuations, which for a strictly one-dimensional system with short-range interactions would preclude the occurrence of a phase transition at finite temperature. Nonvanishing interchain coupling as an effect of three dimensionality in quasi-one-dimensional systems partly suppresses the influence of fluctuations. The theory taking both effects into account shows nevertheless that the mean-field description gives at least qualitative insight. ${ }^{8}$ At low temperatures fluctuations decrease, and quantum effects become negligible due to the high mass of the CDW condensate. Therefore the Peierls gap of a real system at zero temperature will be nearly the same as predicted by mean-field theory, but on the other hand the transition occurs below the mean-field value:

$$
\Delta(0) \approx \Delta_{\mathrm{MF}}(0) \quad T_{P}^{*}<T_{P}^{\mathrm{MF}} .
$$

Following Ref. 4, it is assumed now from (A5) that the actual dependence is a scaled mean-field dependence

$$
\Delta(T) \approx \Delta_{\mathrm{MF}}\left(T \frac{T_{P}^{\mathrm{MF}}}{T_{P}^{*}}\right),
$$

which now contains two independent parameters $\Delta(0)$ and $T_{P}^{*} . \Delta(0)$ is determined from the slope of the strictly linear dependence of $\ln \left(\sigma_{\|}\right)$vs $T^{-1}$ at low temperatures $(50 \leq T \leq 100 \mathrm{~K})$ as seen in Fig. 1. To determine $T_{P}^{*}$ one also has to consider the pseudogap in the electronic density of states above the transition. The latter is a direct consequence of the fluctuations for sufficiently weak interchain coupling, as shown in Ref. 6. In the pseudogap the density of states near the Fermi energy is small but finite. To model the influence of this fluctuating pseudogap, it was replaced in Ref. 9 by an effective uniform and temperature-dependent energy gap $2 \Delta_{\text {eff }}(T)$, with corresponding modification of the one-dimensional density of states below and above the transition. The temperaturedependence $\Delta_{\text {eff }}(T)$ is determined by the activation energy $\Delta(0)$, and, using the connection ${ }^{9}$

$$
\frac{\chi^{\text {spin }}}{\chi^{\text {Pauli }}}=\int_{\Delta_{\mathrm{eff}} / k_{B} T}^{\infty} \frac{x}{\sqrt{x^{2}-\left(\Delta_{\mathrm{eff}} / k_{B} T\right)^{2}}} \frac{e^{x}}{\left(e^{x}+1\right)^{2}} d x,
$$

from the measured paramagnetic spin susceptibility ${ }^{5}$ leading to the dependence shown in Fig. 2. Finally, one can determine $T_{P}^{*}$ by requiring that at some temperature $T_{P}$ the scaled mean-field gap and the pseudogap are equal: $\Delta\left(T_{P}\right)=\Delta_{\text {eff }}\left(T_{P}\right)$. Then $T_{P}$ is the temperature of the transition seen in the derivative of the conductivity data. The resulting overall $T$ dependence of the gap is shown in Fig. 2.

\section{APPENDIX B}

A fundamental criterion for the applicability of kinetic equations like the Boltzmann equation is ${ }^{21}$

$$
\bar{\tau} \gg \hbar / E \approx \hbar / k_{B} T
$$

(the second expression for nondegenerate systems), expressing simply that the energy uncertainty must be sufficiently small for scattering to be elastic. It seems to be worthwhile to express these conditions in the following form:

$$
\begin{aligned}
& \bar{\tau} \gg 2.6 \times 10^{-14} \mathrm{~s}\left(\frac{300 \mathrm{~K}}{T}\right), \\
& \mu \gg 45 \frac{\mathrm{cm}^{2}}{\mathrm{~V} s}\left[\frac{300 \mathrm{~K}}{T} \frac{m}{m_{\mathrm{eff}}}\right), \\
& \lambda \gg 1.7 \mathrm{~nm}\left(\frac{300 \mathrm{~K}}{T} \frac{m}{m_{\mathrm{eff}}}\right)^{1 / 2},
\end{aligned}
$$

which in the cases of the mobility and mean free path depend also on the effective mass. Fitting data with expressions basically following from kinetic equations should be accompanied by checking whether these conditions are fulfilled. 
${ }^{1}$ D. Jérome and H. J. Schulz, Adv. Phys. 31, 299 (1982); K. Bechgaard and D. Jérome, Phys. Scr. T 39, 37 (1991).

${ }^{2}$ S. Kagoshima, H. Nagasawa, and T. Sambongi, OneDimensional Conductors (Springer, Berlin, 1988).

${ }^{3}$ For a review of CDW conductors see, for example, Electronic Properties of Inorganic Quasi-One-Dimensional Compounds $I+I I$, edited by P. Monceau (Reidel, Dordrecht, 1985); Charge Density Waves in Solids, edited by L. P. Gorkov and G. Grüner (North-Holland, Amsterdam, 1989).

${ }^{4}$ W. Brütting, W. Riess, and M. Schwoerer, Ann. Phys. 1, 409 (1992).

${ }^{5}$ U. Köbler, J. Gmeiner, and E. Dormann, J. Magn. Magn. Mater. 69, 189 (1987).

${ }^{6}$ P. A. Lee, T. M. Rice, and P. W. Anderson, Phys. Rev. Lett. 31, 462 (1973).

${ }^{7}$ M. J. Rice and S. Strässler, Solid State Commun. 13, 1389 (1973).

${ }^{8}$ H. J. Schulz in, Low-Dimensional Conductors and Superconductors, edited by D. Jérome and L. G. Caron (Plenum, New York, 1987), p. 95.

${ }^{9}$ D. C. Johnston, Phys. Rev. Lett. 52, 2049 (1984).

${ }^{10}$ V. Enkelmann, B. S. Morra, Ch. Kröhnke, G. Wegner, and J. Heinze, Chem. Phys. 66, 303 (1982).

${ }^{11}$ Th. Schimmel, B. Koch, H. P. Geserich, and M. Schwoerer, Synth. Met. 33, 311 (1989).

${ }^{12}$ V. Ilakovac, S. Ravy, J. P. Pouget, W. Riess, W. Brütting, and
M. Schwoerer, J. Phys. (Paris) C2, 137 (1993).

${ }^{13}$ W. Riess, W. Schmid, J. Gmeiner, and M. Schwoerer, Synth. Met. 42, 2261 (1991).

${ }^{14}$ W. Riess, W. Brütting, and M. Schwoerer, Mol. Cryst. Liq. Cryst. 230, 89 (1993).

${ }^{15}$ W. Riess, W. Brütting, and M. Schwoerer, Synth Met. 55-57, 2664 (1993).

${ }^{16} \mathrm{C}$. Hauenschild, H. W. Helberg, W. Riess, W. Brütting, and M. Schwoerer, Synth. Met. 55-57, 2635 (1993).

${ }^{17}$ Th. Schimmel, W. Riess, G. Denninger, and M. Schwoerer, Ber. Bunsenges. Phys. Chem. 91, 901 (1987).

${ }^{18} \mathrm{M}$. Mehring, in Low-Dimensional Conductors and Superconductors (Ref. 8), p. 185.

${ }^{19}$ D. C. Johnston, J. P. Stokes, and R. A. Klemm, J. Magn. Magn. Mater 54-57, 1317 (1986).

${ }^{20}$ S. Kivelson and A. J. Heeger, Synth. Met. 22, 371 (1988).

${ }^{21}$ V. L. Bronch-Brujevich and S. G. Kalashnikov, Fizika Poluprovodnikov (Nauka, Moscow, 1977) Chap. XIII.

${ }^{22}$ E. M. Conwell in Highly Conducting Quasi-One-Dimensional Organic Crystals, edited by R. K Willardson and A. C. Beer, Semiconductors and Semimetals Vol. 27, (Academic, Boston, 1988), p. 215.

${ }^{23}$ J. M. Ziman, Principles of the Theory of Solids (Cambridge University Press, London, 1972).

${ }^{24}$ L. Pietronero, Synth Met. 8, 225 (1983).

${ }^{25}$ G. A. Toombs, Phys. Rep. 40, 181 (1978). 